\title{
RESIDUAL STRESS MEASUREMENT IN YBCO THIN FILMS*
}

Jae Hong Cheon and J. P. Singh

Energy Technology Division

Argonne National Laboratory

Argonne, IL 60439

April 2002

The submitted manuscript has been created by the
University of Chicago as Operator of Argonne
National Laboratory ("Argonne") under Contract No.
W-31-109-ENG-38 with the U.S. Department of
Energy. The U.S. Government retains for itself, and
others acting on its behalf, a paid-up, nonexclusive,
irrevocable worldwide license in said article to
reproduce, prepare derivative works, distribute copies
to the public, and perform publicly and display
publicly, by or on behalf of the Government.


To be submitted for the Proceedings of the Focused Session on High Temperature Superconductor Processing, $104^{\text {th }}$ Annual Meeting and Exposition of the American Ceramic Society, St. Louis, MO, April 28-May 1, 2002.

* Work supported by the U.S. Department of Energy (DOE), Energy Efficiency and Renewable Energy, as a part of a DOE program to develop electric power technology, under Contract W-31-109-Eng-38.

\section{RESIDUAL STRESS MEASUREMENT IN YBCO THIN FILMS*}

Jae Hong Cheon and J. P. Singh

Energy Technology Division

Argonne National Laboratory

Argonne, IL 60439

\section{ABSTRACT}

Residual stress in YBCO films on Ag and Hastelloy $\mathrm{C}$ substrates was determined by using 3-D optical interferometry and laser scanning to measure the change in curvature radius before and after film deposition. The residual stress was obtained by appropriate analysis of curvature measurements. Consistent with residual thermal stress calculations based on the thermal expansion coefficient mismatch between the substrates and YBCO film, the measured residual stress in the YBCO film on Hastelloy $\mathrm{C}$ substrate was tensile, while it was compressive on the Ag substrate. The stress values measured by the two techniques were generally in good agreement, suggesting that optical 
interferometry and laser scanning have promise for measuring residual stresses in thin films.

\section{INTRODUCTION}

In recent years, substantial effort has been concentrated on processing Y-Ba-Cu-O (YBCO)-based coated conductors by various techniques because of their potential to provide improved electrical properties in magnetic fields [1,2]. Typical processing approaches include ion-beam-assisted deposition, rolling-assisted biaxially textured substrate method, pulsed laser deposition (PLD), e-beam co-evaporation, and metal organic deposition [3-7]. In a coated conductor system, a metallic or ceramic substrate is coated with a ceramic superconductor (i.e., YBCO) with buffer layers in between that act as diffusion barriers between the substrate and YBCO film. Various substrates such as $\mathrm{LaAlO}_{3}$, Hatelloy $\mathrm{C}$, nickel, and silver (Ag) have been used to process coated conductors. Because the various layers in coated conductors have different expansion coefficients, residual strains (and hence stresses) develop in different layers during processing. These stresses may cause microcracking and damage of the superconductor layer, resulting in degradation of superconducting properties and reduction in service life. Therefore, it is critical to evaluate residual stresses in coated conductors and understand the role of various processing parameters that influence these stresses.

Several techniques can be used to measure residual stresses in various thin layers (films) of coated conductors. X-ray diffraction [8] has been used to measure residual stresses in films by determining the change in lattice spacing due to the stresses. In addition, 
the curvature measurement technique [9] has been used to measure residual stresses in films by evaluating the change of the radius of curvature of the film as a result of the stresses. In this study, we determined the residual stresses in YBCO films on Ag and Hastelloy C substrates by a curvature measurement technique using 3-D optical interferometry and laser scanning.

\section{MATERIALS AND METHODS}

Coated conductors using YBCO films on Ag and Hastelloy C substrates were processed by the PLD technique. The substrates $(1.5 \mathrm{~cm} \times 1.2 \mathrm{~cm})$ were polished to an approximately $1 \mu \mathrm{m}$ finish on both sides and were subsequently subjected to an ultrasonic cleaning to obtain flat and clean surfaces. The substrate thickness (ranging from $30 \mu \mathrm{m}$ to $200 \mu \mathrm{m}$ ) was measured by scanning electron microscopy.

The PLD process was carried out with a Lambda Physik COMPex 201 Excimer laser having a $\mathrm{Kr}-\mathrm{F}_{2}$ gas mixture as the laser medium [10]. The substrates were placed on a rotating inclined sample holder. The samples were glued to the holder with a silver paste and were heated to $700-800^{\circ} \mathrm{C}$ during the deposition process. A commercial YBCO target ( 1 inch in diameter and 0.25 inch thick) was used and the distance from target to substrate was maintained at $4-8 \mathrm{~cm}$. The oxygen partial pressure in the deposition chamber was maintained in the range of 100-300 mTorr by flowing ultrahigh-purity oxygen through the chamber. The energy density for deposition was estimated to be $\approx 1-3 \mathrm{~J} / \mathrm{cm}^{2}$. The thickness of the deposited YBCO films was measured 
to be $\approx 300 \mathrm{~nm}$. Four PLD specimens were prepared for each substrate. The details of the deposition process are described in Reference [10].

Residual stresses in the YBCO films on Ag and Hastelloy C substrates were evaluated by measuring the change in the radius of curvature of the substrates before and after the film deposition. It was assumed that the curvature change in substrate also represents the change in curvature of the film in the absence of film delamination. The radius of curvature was measured by 3-D optical interferometric surface mapping [11] and laser scanning [12]. For both the optical and laser scanning methods, three to five curvature measurements in the $\mathrm{x}$ - and $\mathrm{y}$-directions were made for each specimen (Fig. 1).

In the optical interferometric technique, the curvature of the film was determined by counting the number of interference fringes [13]. In the laser scanning technique, a laser beam was reflected off the curved surfaces of the coated substrate specimens. A rotating mirror guided the reflected laser beam to scan the curved surface in the desired direction. A position-sensitive photodetector determined the intensity change in the reflected beams from different locations resulting from the surface curvature [13]. The radius of curvature $(\mathrm{R})$ was obtained from the gradient $(\kappa)$ of the plot representing laser detection intensity as a function of mirror position (Fig. 2). With the measured value of $\kappa$, the radius of curvature was calculated from Equation 1 [12].

$$
\mathrm{R}=2 /(0.36442 \kappa)
$$


Subsequently, the residual stress $(\sigma)$ in the YBCO film was estimated from Equation 2 [14].

$$
\sigma=\mathrm{E}_{\mathrm{f}} \mathrm{t}_{\mathrm{s}}^{2} /\left(6 \Delta \mathrm{Rt}_{\mathrm{f}}\right)
$$

In Equation 2, $E_{f}=E /(1-v)$ is the bi-axial Young's modulus of elasticity, where $E$ is the Young's modulus of elasticity, and $v$ is Poisson's ratio. Also, $\Delta \mathrm{R}$ is the difference in the radius of curvature of the film (or the substrate) before and after the film deposition, $t_{s}$ is the substrate thickness, and $t_{f}$ is the film thickness. The thicknesses of the substrate and film were measured by scanning electron microscopy, and the elastic moduli were taken from the literature (Table 1) $[15,16]$.

\section{RESULTS AND DISCUSSION}

The optical interferometric images in Fig. 3 show the curvature of the two substrates before and after YBCO deposition. These images were constructed by computer based on an interference fringe analysis and were subsequently used to determine the radius of curvature of different substrates before and after the YBCO film deposition. Table 2 shows the radii of curvature measured by optical inferometric and laser scanning techniques for Hastelloy $\mathrm{C}$ and $\mathrm{Ag}$ substrates before and after deposition of YBCO film. From Table 2, the change in the radius of curvature $(\Delta \mathrm{R})$ after film deposition was derived for the different substrates and techniques. The residual stresses in the YBCO film were then estimated by substituting the measured values of $\Delta R, t_{s}$, and $t_{f}$ into 
Equation 2. These measured stresses are plotted in Fig. 4. We also estimated the residual stresses $(\sigma)$ due to the thermal expansion mismatch by analytical approach, using Equation 3 [17].

$$
\sigma=\frac{E}{(1-v)} \Delta \alpha \Delta T
$$

where E and $v$ are the elastic modulus and Poisson's ratio of the film, respectively; $\Delta \alpha$ is the difference in thermal expansion coefficients of the substrate and the film; and $\Delta \mathrm{T}$ is the difference between the room and processing temperature. For comparison, these calculated values of the residual stresses are shown in Figure 4.

As seen in Figure 4, there is a reasonably good agreement between the stress values measured by the optical and laser techniques. The residual stresses in the YBCO film on Hastelloy $\mathrm{C}$ substrate were measured to be 47 and $76 \mathrm{MPa}$ by the optical and laser methods, respectively. The corresponding stresses in the YBCO film on Ag substrate were measured to be 1024 and $1078 \mathrm{MPa}$. However, these measured values differed from the residual stresses calculated from the thermal expansion coefficient mismatch with Equation 3: $160 \mathrm{MPa}$ for Hastelloy $\mathrm{C}$ substrate and $882 \mathrm{MPa}$ for Ag substrate.

We reached three important conclusions from our results. First, the residual stresses in YBCO film on Hastelloy $\mathrm{C}$ substrate are tensile, while the stresses are compressive for the Ag substrate. This is inferred from the expansion coefficient of YBCO being larger 
than that of Hastelloy $\mathrm{C}$ and smaller than that of Ag (Table 1). Second, the measured tensile stress in the YBCO film on Hastelloy $\mathrm{C}$ substrate is slightly smaller than the calculated value, whereas the measured compressive stress in the film on Ag substrate is slightly larger than the calculated value (Fig. 4). This difference is believed to be due to the presence of an internal compressive stress that developed during the PLD processing due to Ar ion-peening effects. Verification of this hypothesis is underway. Third, the residual stresses measured by the two techniques with the same substrate are the same order of magnitude (Fig. 4), indicating that these techniques show considerable promise for stress measurements in thin YBCO films.

\section{ACKNOWLEDGMENT}

This work supported by the U.S. Department of Energy (DOE), Energy Efficiency and Renewable Energy, as a part of a DOE program to develop electric power technology, under Contract W-31-109-Eng-38. We thank Meiya Li and Beihai Ma for providing coated-conductor specimens with YBCO film.

\section{REFERENCES}

1. A. Goyal et al., Appl. Phys. Lett. 69 (1996) 1795.

2. D. P. Norton et al., Science 274 (1996) 755.

3. Y. Iijima, N. Tanabe, O. Kono, and Y. Ikeno, Appl. Phys. Lett. 60 (1992) 759. 
4. H. Qing, D. K. Christen, J. D. Budai, E. D. Specht, D. F. Lee, A. Goyal, D. P. Norton, M. Paranthaman, F. A. List, and D. M. Kroeger, Physica C 275 (1997) 155.

5. K. Fujino, N. Yoshida, N. Hayashi, S. Okuda, T. Hara, and H. Ishii, Advances in Superconductivity VI, Springer, Tokyo, 1994, p. 763.

6. H. Kim, J. Yoo, K. Jung, J. Lee, S. Oh, and D. Youm, Supercond. Sci. Technol. 13 (2000) 995 .

7. P. C. McIntyre, M. J . Cima, and A. Roshkon, J. Appl. Phys. 77 (1995) 5263.

8. H. P. Klug and L. E. Alexander, X-ray Diffraction Procedures for Polycrystalline and Amorphous Materials, $2^{\text {nd }}$ edn, Wiley, New York, 1974, ch. 11.

9. P. A. Flinn, MRS Proceedings 130 (1989) 41.

10. B. Ma, M. Li, Y. A. Jee, R. E. Koritala, B. L. Fisher, and U. Balachandran Physica C 366 (2002) 270.

11. R. E. Cuthrell, D.M. Mattox, C. R. Peeples, P.L. Dreike, and K.P. Lamppa, J. Vac. Sci. Technol., A 6 (1988) 2914.

12. P. A. Flinn, D. S. Gardner, and W. D. Nix, IEEE Transactions on Electronic Devices 34 (1987) 689 .

13. W. D. Nix, Metallurgical Transactions A 20A (1989) 2217.

14. G. G. Stoney, Proceedings of the Royal Society, London, Series A 82 (1909) 172.

15. Goodfellow Cambridge Limited, Huntingdon, England, "Technical Data : Ag and Hastelloy C" www.goodfellow.com (2002).

16. A. S. Raynes, S. W. Freiman, F. W. Gayle, and D. L. Kaiser, Journal of Applied Physics 70 (1991) 5254.

17. D. Burgreen, Elements of Thermal Stress Analysis, C. P. Press, New York, 1971, p. 462. 

Table 1. Mechanical and physical properties of Hastelloy and Ag substrates and YBCO

\begin{tabular}{|l|c|c|c|}
\hline & Hastelloy $\mathrm{C}^{15}$ & $\mathrm{Ag}^{15}$ & $\mathrm{YBCO}^{16}$ \\
\hline Melting Temperature $\left({ }^{\circ} \mathrm{C}\right)$ & 1300 & 961.9 & 1270 \\
\hline Thermal Expansion Coefficient $\left(10^{-6} \mathrm{~K}^{-1}\right)$ & 12.5 & 19.4 & 13.4 \\
\hline Elastic Modulus $(\mathrm{GPa})$ & $170-220$ & 100 & $135-157$ \\
\hline
\end{tabular}

Table 2. Radius of curvature measured by optical interferometric and laser scanning methods for Hastelloy and Ag substrates before and after deposition of YBCO film.

\begin{tabular}{|l|c|c|c|c|}
\hline \multirow{2}{*}{} & \multicolumn{2}{|c|}{ Optical Interferometry } & \multicolumn{2}{c|}{ Laser Scanning } \\
\cline { 2 - 5 } & Hastelloy C & $\mathrm{Ag}$ & Hastelloy C & $\mathrm{Ag}$ \\
\hline $\begin{array}{l}\text { Radius of Curvature } \\
\text { before Deposition }(\mu \mathrm{m})\end{array}$ & $2.07 \mathrm{e}+05$ & $4.92 \mathrm{e}+05$ & $2.18 \mathrm{e}+05$ & $5.12 \mathrm{e}+05$ \\
\hline $\begin{array}{l}\text { Radius of Curvature } \\
\text { after Deposition }(\mu \mathrm{m})\end{array}$ & $2.06 \mathrm{e}+05$ & $6.02 \mathrm{e}+05$ & $2.16 \mathrm{e}+05$ & $6.46 \mathrm{e}+05$ \\
\hline
\end{tabular}




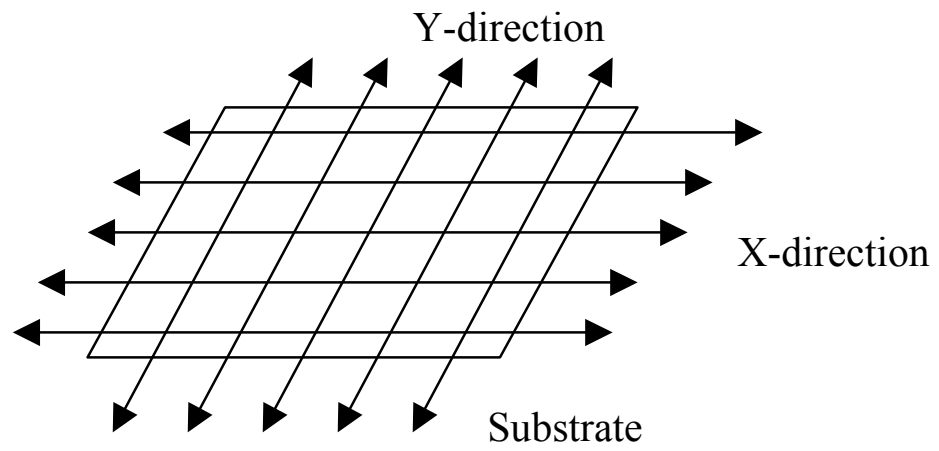

Fig. 1. Schematic showing $\mathrm{x}$ - and $\mathrm{y}$-directions on the measured curved substrates.

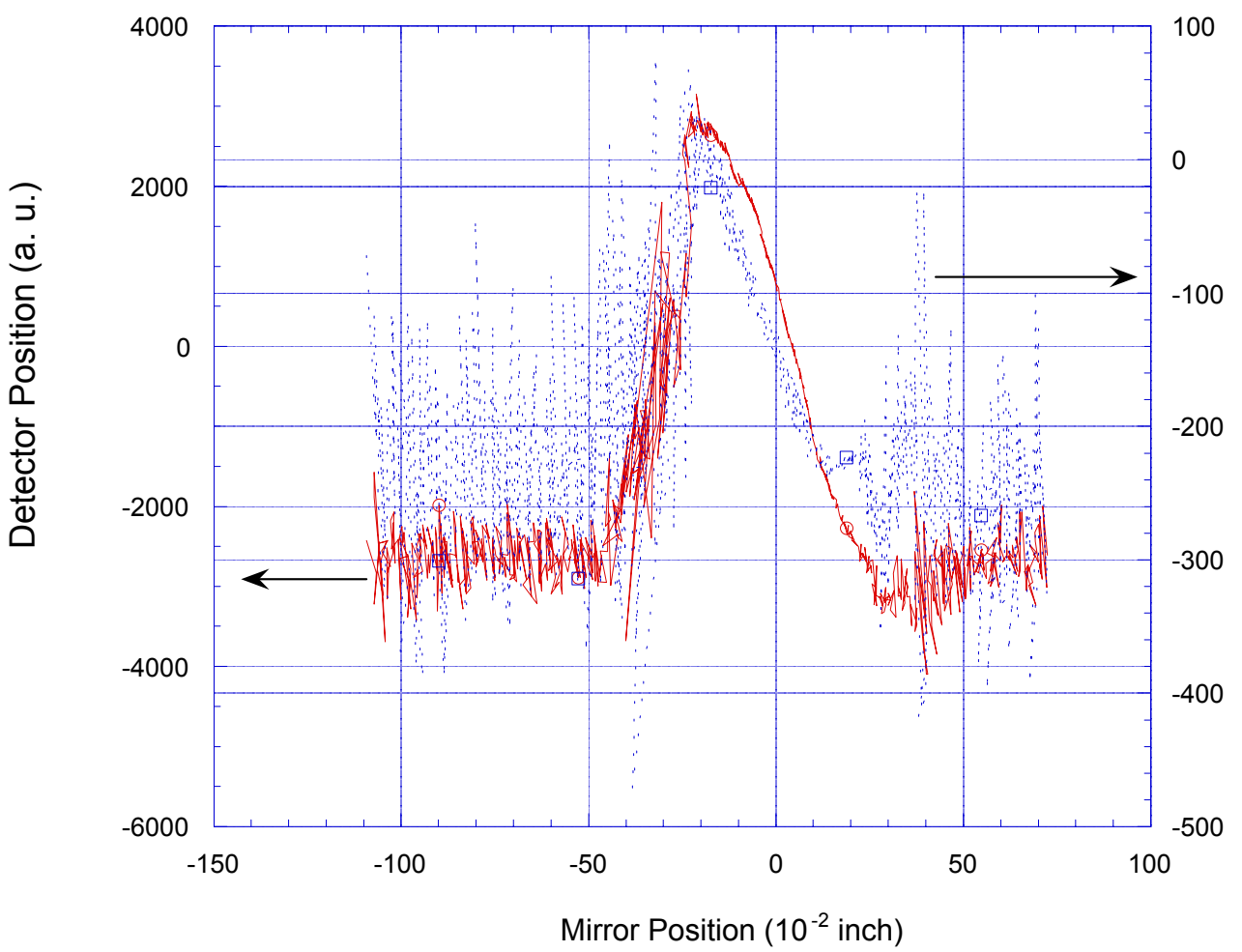

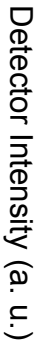


Fig. 2. Typical variation of laser detection intensity as a function of mirror position.

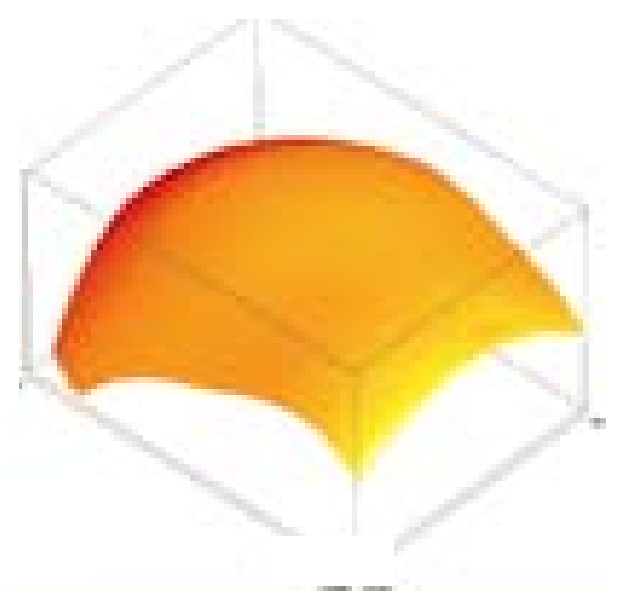

Before Deposition

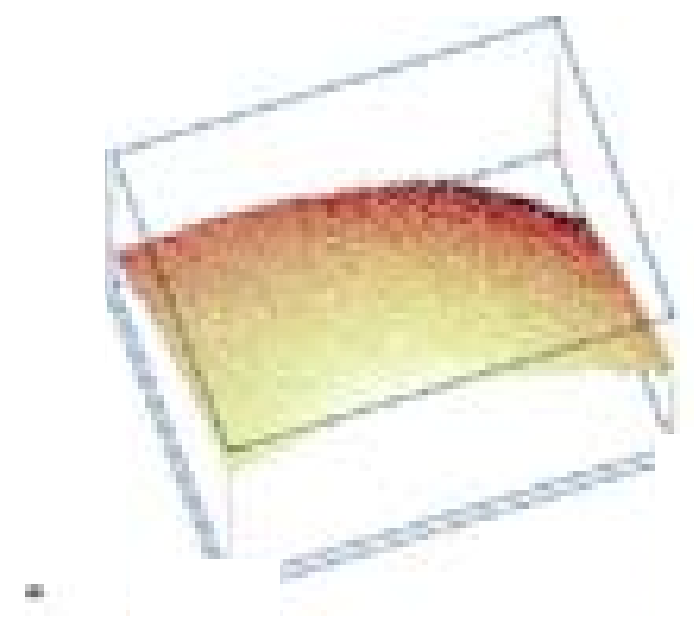

Before Deposition

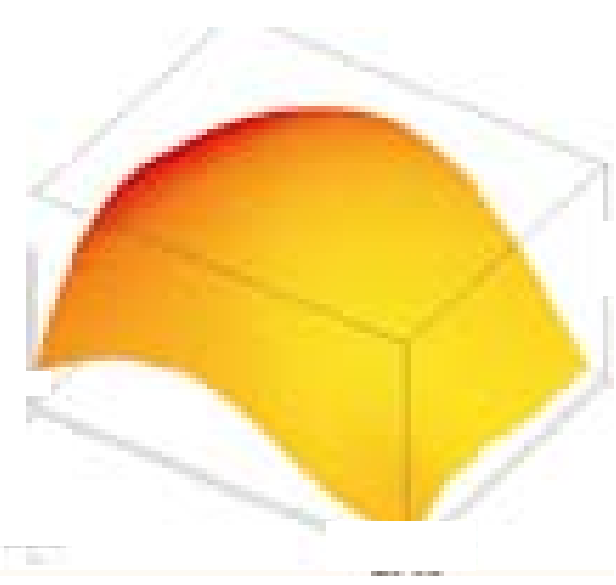

(a) Hastelloy C
After Deposition

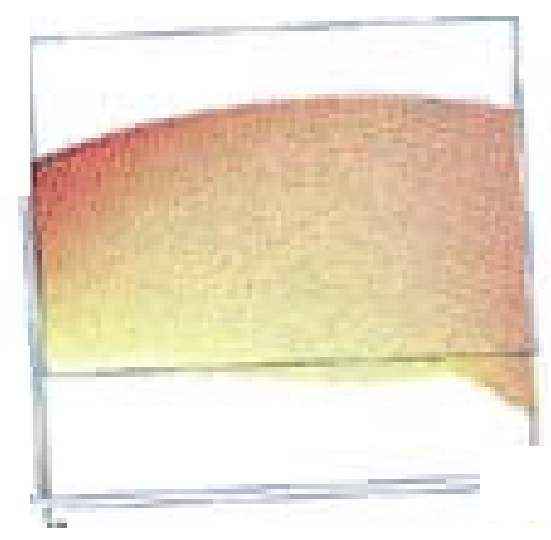

(b) Ag

After Deposition 
Fig. 3. Optical interferometric images showing the curvature of substrates before and after YBCO deposition on (a) Hastelloy C and (b) Ag substrates.

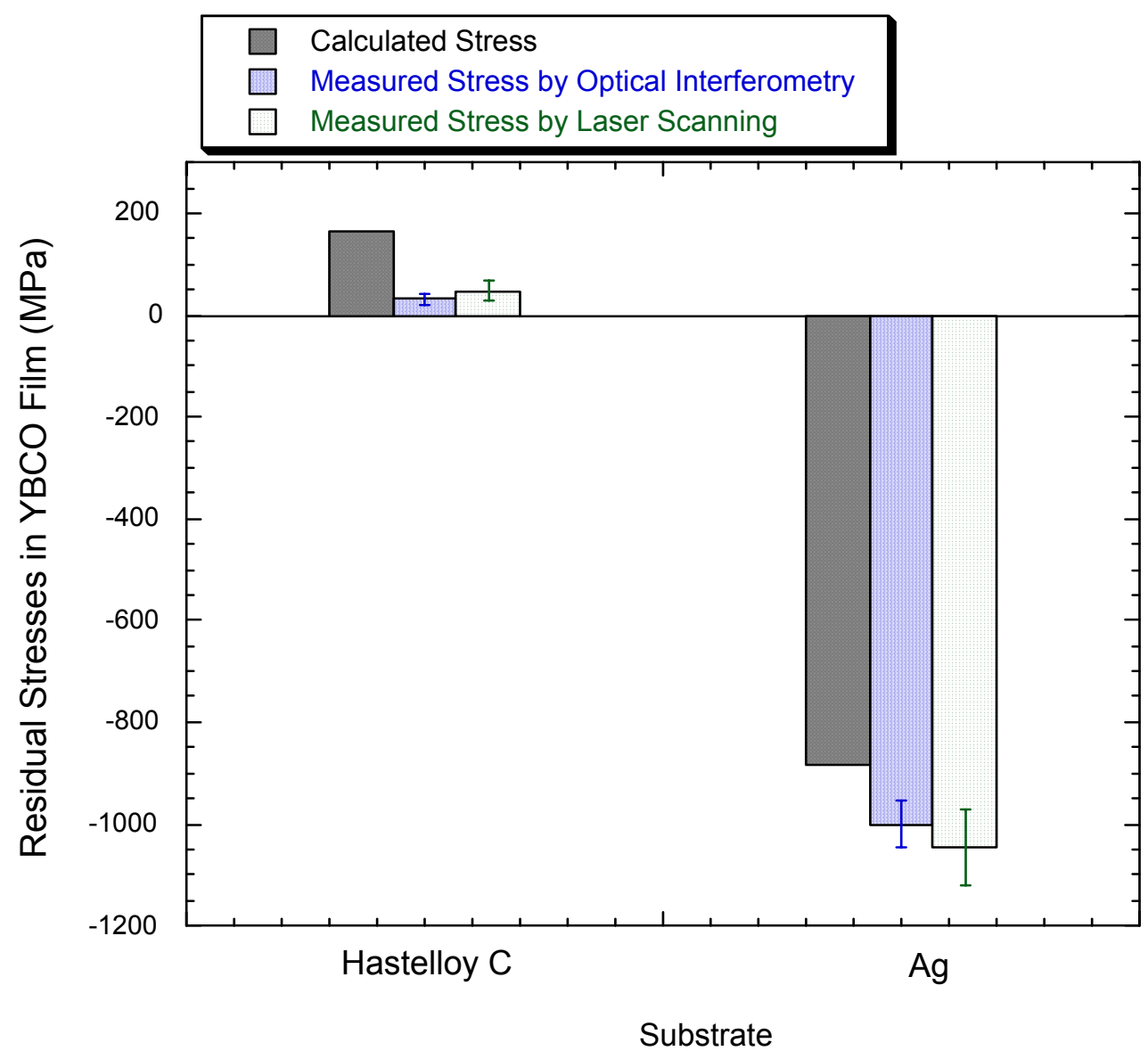

Fig. 4. The measured and calculated (based on thermal mismatch) residual stresses in YBCO films on Hastelloy C and Ag substrates. 